\title{
Splenogonadal fusion and testicular cancer: case report and review of the literature
}

\author{
Fusão esplenogonadal e câncer testicular: relato de caso e revisão da literatura
}

\author{
Roberto Iglesias Lopes ${ }^{1}$, Mabel Tatty de Medeiros ${ }^{2}$, Marco Antônio Arap³, Marcello Cocuzza ${ }^{4}$, \\ Miguel Srougi ${ }^{5}$, Jorge Hallak ${ }^{6}$
}

\begin{abstract}
A 36 year-old man after tests for assessing male infertility was diagnosed with primary infertility, bilateral cryptorchidism, nonobstructive azoospermia and discontinuous splenogonadal fusion. Carcinoma in situ was found in his left testicle, which was intraabdominal and associated with splenogonadal fusion. To our knowledge, this is the fourth case of splenogonadal fusion associated with testicular cancer reported. One should always bear in mind the possibility of this association for the left cryptorchid testicle.
\end{abstract}

Keywords: Infertility, male; Azoospermia; Crypthorchidism; Testicular neoplasms; Spleen/abnormalities; Testis/abnormalities; Laparoscopy; Microdissection; Magnetic resonance imaging; Case reports

\section{RESUMO}

Um homem de 36 anos, depois de ser submetido a exames para avaliação de infertilidade masculina, foi diagnosticado com infertilidade masculina primária, criptorquidia bilateral, azoospermia não obstrutiva e fusão esplenogonadal descontínua. Carcinoma in situ estava presente no testículo esquerdo, que tinha localização intra-abdominal e estava associado à fusão esplenogonadal. Esse é o quarto caso de fusão esplenogonadal associada a câncer testicular, segundo nossa avaliação. Deve-se sempre ter em mente a possibilidade dessa associação em testículos criptorquídicos à esquerda.

Descritores: Infertilidade masculina; Azoospermia; Criptorquidismo; Neoplasias testiculares; Baço/anormalidades; Testículos/anormalidades; Laparoscopia; Microdissecção; Imagem por ressonância magnética; Relatos de casos

\section{INTRODUCTION}

Splenogonadal fusion is a rare congenital anomaly that interferes with normal gonadal descent and closure of the processus vaginalis due toan abnormal attachment of the splenic tissue to the gonad, which can be in a continuous or discontinuous manner. As a result, this is associated with cryptorchidism and inguinal hernia ${ }^{(1)}$.

Cryptorchidism is a well-known cause of infertility due to spermatogenesis alteration by the exposure of the undescended testis to high temperatures that combined with hormonal imbalances as androgens and insulinlike factors has an important role in gubernaculum development, and in the subsequent testicular migration, that may finally result in severe oligozoospermia or azoospermia $^{(2)}$.

It is also a recognized condition associated with an increased incidence of testicular cancer with an estimated incidence of $1.7 \%$ for carcinoma in situ ${ }^{(2)}$.

Malignancy associated with splenogonadal fusion is exceedingly rare and, to our knowledge, only three cases of this association have been reported in the literature ${ }^{(3)}$.We report a case of testicular cancer with concurrent presentation of splenogonadal fusion, cryptorquidism, non-obstructive azoospermia and male infertility.

\footnotetext{
${ }_{1}$ Graduate Program (Doctorate degree) in Urology, Faculdade de Medicina, Universidade de São Paulo - USP, São Paulo (SP), Brazil.

${ }^{2}$ Patology Division, Hospital das Clínicas, Faculdade de Medicina, Universidade de São Paulo - USP, São Paulo (SP), Brazil.

${ }^{3}$ Laparoscopy Team, Hospital das Clínicas, Faculdade de Medicina, Universidade de São Paulo - USP, São Paulo (SP), Brazil.

${ }^{4}$ Infertility Team, Hospital das Clínicas, Faculdade de Medicina, Universidade de São Paulo - USP, São Paulo (SP), Brazil.

${ }^{5}$ Department of Urology, Faculdade de Medicina, Universidade de São Paulo - USP, São Paulo (SP), Brazil.

${ }^{6}$ Infertility Team, Hospital das Clínicas, Faculdade de Medicina, Universidade de São Paulo - USP, São Paulo (SP), Brazil.

Corresponding author: Roberto Iglesias Lopes - Roberto Iglesias Lopes - Divisão de Clínica Urológica do Hospital das Clínicas da Faculdade de Medicina da Universidade de São Paulo - Avenida Enéas de Carvalho Aguiar, 255, 70 andar - Zip code: 05403-000 - São Paulo (SP), Brazil - Phone: (11) 3669-8080 - E-mail: robertoiglesias@terra.com.br

Received on: Jan 26, 2011 - Accepted on: Jan 19, 2012
} 


\section{CASE REPORT}

A 36-year-old man presented with 8 years of primary infertility. His wifewas 28 years old, had regular cycles, and normal initial infertility evaluation, including a hysterosalpingogramdone previously to her partner initial evaluation. Previous surgical history included a right orchidopexy for inguinal testis when he was 31 years old. The patient had normal secondary sexual characters, an atrophic right testis positioned above the external inguinal ring and a non-palpable left testis. Hormonal evaluation included an $\mathrm{FSH}=22 \mathrm{IU} / \mathrm{L}$ (normal: $<10.5 \mathrm{IU} / \mathrm{L}$ ), $\mathrm{LH}=10.7 \mathrm{IU} / \mathrm{L}$ (normal: 1.0 to $8.4 \mathrm{IU} / \mathrm{L}$ ), total testosterone level of $221 \mathrm{ng} / \mathrm{dL}$ (normal: 271 to $965 \mathrm{ng} / \mathrm{dL}$ ). Tumor markers included testicular serum alphafetoprotein $=5.8 \mathrm{ng} / \mathrm{mL}$ (normal range: $<10 \mathrm{ng} / \mathrm{mL}$ ) and chorionic gonadotrophin $<3$ IU (normal value in men: $<3$ UL).

Evaluation of the ejaculate included two complete semen analyses with centrifugation and cytocentrifugation to detect sperm in the ejaculate. We used the Cytospin 3 centrifuge (Shandon, Pittsburg, USA)and stained slides by NF-PICS (nuclear fast red \#1159390025, Merck, Germany, indigocarmine \#1047410025, Merck, Germany). A careful search for sperm, which nucleus stained in pink, was also performed in 100x by immersion oil objective. Both semen analyses revealed total azoospermia.The genetic testing revealed a normal karyotype $(46, \mathrm{XY})$ and the Y microdeletion testing revealed no abnormalities.

Ultrasonography showed a right testis of $2.5 \times 1.1 \times 1.7 \mathrm{~cm}$ (volume of $2.64 \mathrm{cc}$ ) located on the right inguinal region, but left testis was not identified. Magnetic resonance imaging revealed a left testis located below the spleen (Figure 1). The splenogonadal fusion was considered among the possibilities because the spleen and the gonad are embryologically related in the early stages of organs development.

We decided to perform a left orchiectomy combined with immediate bench testicular microdissection because this patient had a left atrophic testis with probable embryological attachment alterations associated with azoospermia, due to testicular dysgenesis, and a statistically higher risk of testicular malignancy. The bench testicular microdissection was made to search for viable sperm for cryopreservation and future use in assisted reproductive techniques as the patient was married, childless and expressed the desire to have his own genetic offspring.

During laparoscopy the left atrophic testis had normal macroscopic appearance, and it was located on the upper border of the psoas muscle, $2 \mathrm{~cm}$ below the inferior splenic pole, with no sites of attachment to the spleen. The vas deferens, which ascended from the pelvis, was normal. We clipped it followed by the clipping of gonadal vessels.

The bench testicular microdissection enabled the collection of multiple samples from all areas of the testis that most likely to have sperm, enlarged seminiferous tubules. After the carefully extraction, samples were searched for the presence of fully formed spermatozoa. Biopsies of the superior, medium and inferior areas of the testis were obtained for histopathological analysis. We found anarea measuring $0.8 \mathrm{~cm}$ of splenogonadal fusion associated with an atrophic testis (Figure 2), and testicular carcinoma in situ with microcalcifications in all testicular parenchyma (Figure 3) in all three microdissection biopsies done. The epidydimis was normal, and no spermatogenesis or spermiogenesis was noted in all fragments.

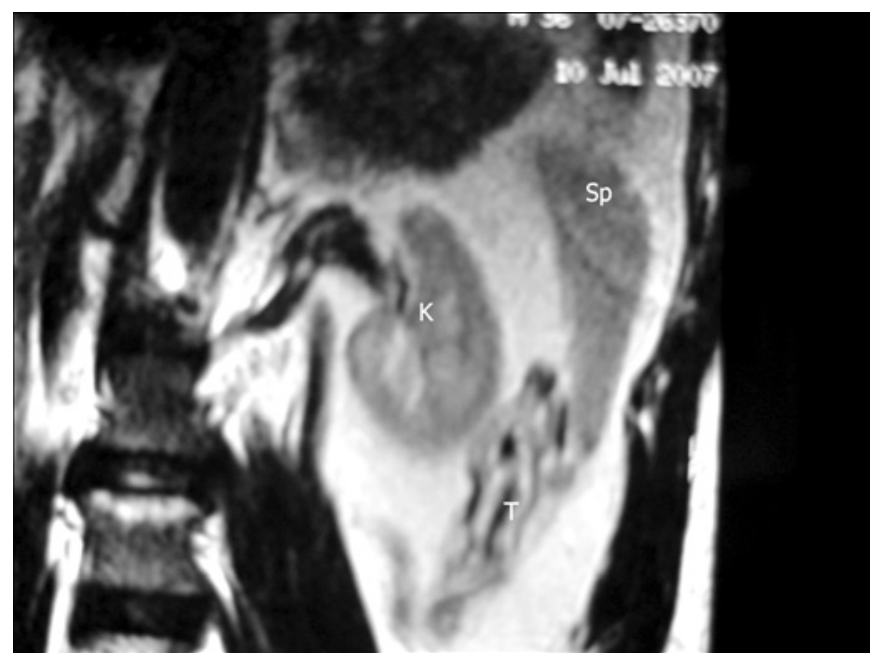

Figure 1. Magnetic resonance showing the left testis $(\mathrm{T})$ below the spleen $(\mathrm{Sp})$; K: kidney

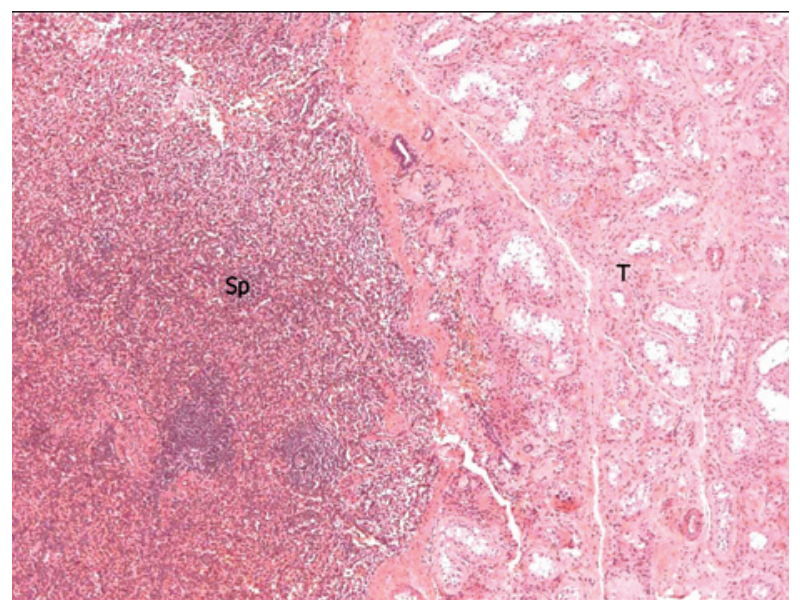

Figure 2. Histopathological examination revealed splenogonadal fusion; Sp: spleen;T: testicular parenchyma 


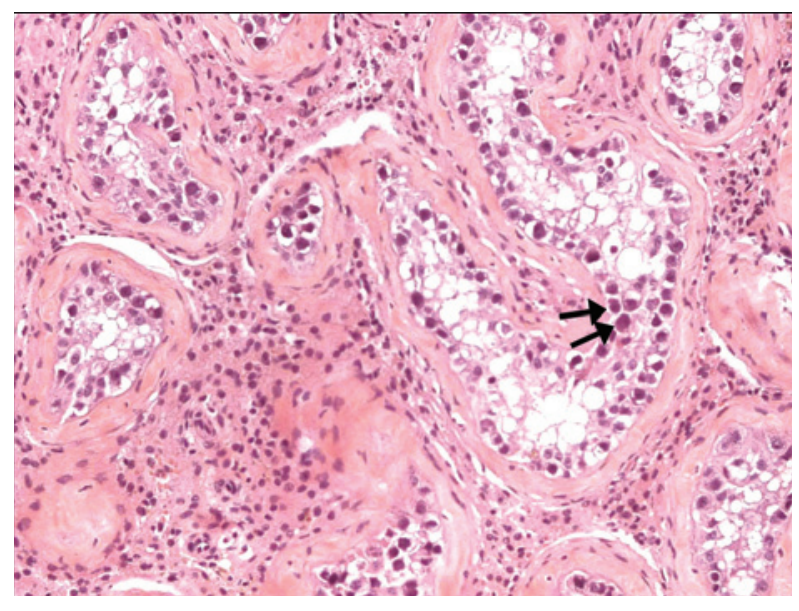

Figure 3. Carcinoma in situ (indicated by the arrows) in testicular parenchyma

In view of the findings on the left side, a right orchidopexy along with biopsies of the upper, mid and lower pole of the testis was scheduled. In the surgery, the testis could be brought only into the external inguinal ring, where it was fixed in place.

The frozen section of testicular biopsy did not reveal carcinoma, and the histopathological analysis showed no spermatogenesis or spermiogenesis, the testis was normal (no carcinoma in situ). Although the right testis was atrophic $(2 \times 1.8 \times 1.8 \mathrm{~cm})$, the patient refused to undergo orchiectomy if testicular cancer was not presented. A left testicular prosthesis was inserted.

\section{DISCUSSION}

Splenogonadal fusion is a rare congenital anomaly with approximately 150 cases reported in the literature since the first description by Bostroem in $1883^{(4)}$. During the 5th week of embryological development the splenic anlage develops in the left dorsal mesogastrium and the spleen comes close to the left urogenital fold during the rotation of the embryonic gut.

The urogenital fold contains the gonadal mesoderm, which develops into gonadal structures. The most accepted theory of splenogonadal fusion is the abnormal attachment that occurs during the 5th to 8th week of gestation when both organs are close ${ }^{(1)}$. Gonadal descent begins on the 8th week and if splenogonadal fusion happens, the splenic tissue attached to the gonad accompanies the gonadal path. Splenogonadal fusion can be in a continuous or in a discontinuous manner as described by Putschar and Manion in 1956(5).

Splenogonadal fusion is usually discovered incidentally in a routine groin exploration for cryptorchidism or hernia ${ }^{(6)}$. In few cases, a hypothesis of splenogonadal fusion can be made preoperatively. In our case, the magnetic resonance to investigate left testis location showed a close relationship between the left testis and the lower pole of spleen. Therefore, we considered splenogonadal fusion a possible diagnosis.

Splenogonadal fusion interferes with closure of processus vaginallis, and it is frequently associated with an ipsilateral inguinal hernia and cryptorchidism ${ }^{(1)}$. Another possible association is micrognathia and limb anomalies $^{(7)}$. In addition, it is more frequent in males, with a male: female ratio of 16.6:1. The vast majority is on the left side (98\% of the cases) ${ }^{(1)}$. Although our patient did not have associated limb abnormalities, he had bilateral cryptorchidia.

Criptorchidism, especially when bilateral, is associated with a higher rate of infertility and azoospermia $^{(8)}$. Regarding cryptorchidism, $80 \%$ of the cryptorchidic testicles can be palpated while $20 \%$ are impalpable(2).

The most common locations for the cryptorchidic testicle are: inguinal canal (63\%), ectopic location (11\%), external inguinal ring (9\%) and abdominal only $2 \%$ (2) When located in an abdominal, the male gonad is exposed to high temperatures which interferes with spermatogenesis and therefore results in azoospermia and infertility ${ }^{(2)}$. Male infertility can be viewed as the end point of a series of serious and complex diseases and syndromes, therefore evaluation for male infertility should be done according to established guidelines, otherwise a tendency to apply excess of assisted reproductive techniques may be a dangerous shortcut $^{(9,10)}$.

Cryptorchidism is a well associated condition with testicular cancer, which has an incidence of $1.7 \%$ of carcinoma in situ, mainly when this is not treated by orchidopexy at early stages of life (2). To our knowledge this report is the fourth case in the literature of testicular malignancy with splenogonadal fusion, cryptorchidism, azoospermia and male infertility.

Splenogonadal fusion alone may not increase the risk of malignancy per se, but the frequent association with cryptorchidism explains the increased risk of testicular cancer. One should always bear in mind the possibility of this association for cryptorchidic testicles.

\section{REFERENCES}

1. Varma DR, Sirineni GR, Rao MV, Pottala KM, Mallipudi BV. Sonographic and CT features of splenogonadal fusion. Pediatr Radiol. 2007;37(9):916-9.

2. Schneck FX, Bellinger MF. Abnormalities of the testis and scrotum and their management. In: Wein AJ, Kavoussi LR, Novick LR, Partin AW, Peters CA, editors. Campbell-Walsh urology. 9th ed. Philadelphia: Saunders Elsevier; 2007 p. 3761-98 
3. Imperial S, Sidhu JS. Nonseminomatous germ cell tumor arising in splenogonadal fusion. Arch Pathol Lab Med. 2002;126(10):1222-5.

4. Boestrom E. Demostration eines praparates von verwachsung der milz mit dem linken hoden. Gellschaft deutscher naturforscher und artze verhandlungen der 56 Versamlung. Freiburg. 1883;149.

5. Putschar WG, Manion WC. Splenic-gonadal fusion. Am J Pathol. 1956;32: 15-35.

6. Ando S, Shimazui T, Hattori K, Yamamoto T, Kuriyagawa K, Akaza H. Splenogonadal fusion: case report and review of published works. Int J Urol. 2006;13(12):1539-41.
7. Duncan Jr WL, Barraza MA. Splenogonadal fusion: a case report and review of the literature. J Pediatr Surg. 2005;40:5-7.

8. Moretti E, Cairano G, Capitani S, Scapigliati G, Baccetti B, Collodel J. Cryptorchidism and semen quality: a TEM and molecular study. J Androl. 2007;28(1):194-9.

9. Bhasin S. Approach to the infertile man. J Clin Endocrinol Metab. 2007; 92(6):1995-2004. Review.

10. Nathanson KL, Kanetsky PA, Hawes R, Vaughn DY, Letrero R, Tucker K, et al. The $Y$ deletion $\mathrm{gr} / \mathrm{gr}$ and susceptibility to testicular germ cell tumor. Am J Hum Genet. 2005;77(6):1034-43. 